\title{
Innovación Social: una experiencia de trabajo colectivo entre la Universidad de Playa Ancha y su territorio ${ }^{1}$
}

\section{Social Innovation: an experience of collective work between the University of Playa Ancha and its territory}

Paula Andrea Retamal Canto**

\section{Resumen}

El presente artículo desarrolla parte del proceso de innovación social llevado a cabo por la Universidad de Playa Ancha a través del Convenio de Desempeño "Un Modelo Replicable de Innovación Social para el Desarrollo Territorial de Playa Ancha". Desde el Observatorio de Innovación Social, se elaboró una Cartografía Socioterritorial del barrio de Playa Ancha, instancia participativa que ha favorecido el diálogo entre actores sociales del territorio y la Universidad, y que ha visibilizado la realidad social del territorio a partir del trabajo colectivo en la identificación de elementos de interés local, rescatando la mirada de los actores sociales respecto al espacio habitado.

\footnotetext{
${ }^{1}$ Antecedentes de este trabajo fueron presentado en el XXXIV Congreso Internacional de la Asociación de Estudios Latinoamericanos (LASA) llevado a cabo en mayo de 2016, en la ciudad de Nueva York, Estados Unidos, con financiamiento del CD UPA 1301 del Ministerio de Educación.

** Licenciada en Sociología y Socióloga de la Universidad de Playa Ancha. Magíster (C en Gestión Cultural de la Universidad de Playa Ancha. Se desempeña como profesional de la Unidad de Innovación Social (UIS) - Dirección General de Vinculación con el Medio, Universidad de Playa Ancha, Valparaíso, Chile. Contacto: paula.retamal@upla.cl
} 


\begin{abstract}
The article develops part of the process social innovation that the University of Playa Ancha has developed through the Performance Agreement "A replicable model of social innovation for territorial development". The University's Social Innovation Observatory carried out a Socioterritorial Mapping of the neighborhood of Playa Ancha through a participatory process that has favored the dialogue between social actors in the territory and the University. Furthermore, this collaborative process has help to visualize the territory's social reality in identifying elements of local interest, rescuing the perspective of the social actors in regards to the place they inhabit.
\end{abstract}

\title{
Palabras clave
}

Espacio habitado, territorio, cartografía social.

\section{Keywords}

Inhabited space, territory, social mapping.

\section{Antecedentes de contexto}

La Universidad de Playa Ancha se adjudicó el año 2013 un Convenio de Desempeño Regional de Educación Superior denominado Convenio de Desempeño UPA 1301: "Generación de conocimiento compartido: Un modelo replicable de Innovación Social para el desarrollo territorial de Playa Ancha", cuyo objetivo central es fortalecer el desarrollo local del territorio y la generación de impactos sociales notables que permitan su replicabilidad en contextos similares. Este Convenio de Desempeño busca lograr una nueva interrelación entre la institución de educación y el territorio, a través de prácticas bidireccionales entre los actores locales y la comunidad universitaria, que promuevan y potencien el intercambio de saberes, necesidades y oportunidades, a partir de la construcción de conocimiento compartido en pro del desarrollo territorial, posicionando el rol de la universidad pública y regional.

La Universidad de Playa Ancha se ubica en la ciudad de Valparaíso, comuna que posee una población estimada de 300.000 habitantes, el 94\% de los cuales habita en los cerros y el $6 \%$ restante se concentra en el borde costero y a pie de cerro (llustre Municipalidad de Valparaíso, 2016). Playa Ancha es el más grande de los 44 cerros de Valparaíso, con una superficie de $6,1 \mathrm{~km}^{2}$ y una población cercana a los 61.469 habitantes (Universidad de Playa Ancha, 2015), representando casi un tercio del total de 
la población. El Convenio de Desempeño ha concentrado sus acciones en este cerro, territorio caracterizado por ser un barrio patrimonial, comercio local, diversas instituciones públicas, alrededor de 450 organizaciones sociales y una activa población universitaria. Playa Ancha es un territorio que reúne una diversidad de actores sociales que se interrelacionan en un espacio con una geografía compleja y heterogénea.

La iniciativa impulsada por el convenio es de innovación social en la medida que busca transformar el territorio social, mediante prácticas y procesos trasformadores sustentables en el tiempo, materializados en una nueva relación entre la Universidad y el territorio. Para esto, se han establecido unidades técnicas para el trabajo territorial e instrumentos que fortalecen el quehacer académico de la institución, fomentando una relación de diálogo con los actores sociales, grupos y organizaciones de Playa Ancha.

Su ejecución comienza en el año 2014, destacándose dos instancias de desarrollo con fuerte enfoque territorial. La primera, la Unidad de Innovación Social (UIS), como área para la gestión y coordinación de instancias de vinculación y relación bidireccional entre la comunidad universitaria y el territorio. Y la segunda, la Mesa Territorial de Desarrollo (MTD), espacio de participación colaborativa entre diversos actores del barrio, representantes de organizaciones sociales (funcionales y territoriales) e institucionales del ámbito local, coordinada por el equipo profesional de la Unidad de Innovación Social.

Estas instancias persiguen el propósito de generar sinergias y compartir saberes, a través de la generación de redes de intercambio y colaboración para mejorar la calidad de vida de los y las habitantes del territorio. Simultáneamente, se apunta a apoyar los procesos formativos de los y las estudiantes de la Universidad de Playa Ancha, mediante prácticas y pasantías en los territorios, haciendo partícipe tanto a la comunidad local como universitaria (académicos/as y estudiantes) de la construcción de conocimiento compartido. Cabe destacar, que con más de dos años de ejecución del Convenio, la Mesa Territorial de Desarrollo ha establecido redes de cooperación y lazos de confianza en este proceso de articulación, bajo el prisma de la innovación social.

En este contexto, la UIS posee dos áreas de gestión para posicionar la vinculación entre la institución y el territorio, fortaleciendo y poniendo en valor procesos de innovación e inclusión social. Estas son la Incubadora Universitaria de Procesos Colaborativos (IUPC) y el Observatorio de Innovación Social (OIS). La incubadora busca la generación de acciones colaborativas para el bienestar colectivo, que surjan a partir del intercambio de saberes entre la Universidad, la Mesa Territorial de Desarrollo y el territorio, desarrollando un diálogo horizontal y colaborativo entre los actores sociales. El Observatorio se instala como una plataforma que monitorea y sistematiza la información como una fuente recopiladora de datos e información, que permite a la comunidad universitaria, especialmente académicos/as y estudiantes, avanzar en la generación de conocimiento, con respecto al territorio y facilitar la toma de decisiones 
en torno a la relación entre la Universidad y las organizaciones sociales, en una relación bi-direccional. Entre sus objetivos se destaca el de sistematizar los ámbitos Socioterritorial, Patrimonial, Convivencia Escolar y Salud, además de las tesis e investigaciones realizadas en el territorio de Playa Ancha en el marco de este Convenio de Desempeño, con el propósito de transferir y difundir conocimientos para el trabajo de académicos/as y estudiantes.

\section{Antecedentes conceptuales sobre espacio y territorio para la elaboración de una cartografía socioterritorial del barrio de Playa Ancha}

La distribución espacial del territorio de Playa Ancha está condicionada no sólo por una dimensión geográfica, característica principal de los cerros de la ciudad de Valparaíso, sino también por procesos históricos y de urbanización que han definido las interrelaciones de los sujetos sociales con el entorno cotidiano, las experiencias del habitar y vivir en el territorio, las prácticas e imaginarios sociales que responden a micro identidades de los y las habitantes de un determinado barrio. De esta forma, existe una realidad heterogénea que caracteriza de una manera especial al cerro Playa Ancha.

Cabe señalar que el poblamiento del cerro Playa Ancha (Universidad de Playa Ancha, 2014) data de mediados del siglo XIX, y fue configurado por familias inglesas de inmigrantes. Sus principales actividades sociales, culturales y comerciales se concentraban en la avenida Gran Bretaña y la calle que bordea la cancha Alejo Barrios, espacios identificados como importantes lugares de encuentro para la vida social de la época. Las familias Waddington, Aguayo y Riofrío fueron quienes comenzaron a realizar los primeros trazados del cerro, imponiendo un estilo arquitectónico inglés en las viviendas de las casas que hoy caracterizan al sector y que poseen una fecha cercana a 1879. Sus habitantes identifican a este sector como la zona histórica y patrimonial de Playa Ancha.

En el año 1906, a partir de la catástrofe que dejó el terremoto en la ciudad de Valparaíso, muchas familias fueron desplazadas hacia el cerro Playa Ancha, lo que incidió en un incremento de la población. En los años 60 y 70, comenzó una importante construcción de viviendas a partir de la Corporación de la Vivienda, que permitió el desarrollo de nuevas poblaciones en el cerro. Asimismo, las tomas de terrenos por familias de escasos recursos significaron una alternativa de hábitat posible en Playa Ancha, situación que en la mayoría de los casos prosperó con la entrega de títulos de dominio. Lo anterior explica cómo la distribución espacial del poblamiento y urbanización del cerro ha estado marcada por coyunturas sociales que, sin duda, se han vinculado con factores socioeconómicos de las familias que se han instalado a vivir en el territorio.

En la medida que el territorio es un "complejo de interconexiones, en donde las relaciones, eventos, fenómenos, dinámicas y procesos son todos recíprocamente 
interdependientes y se retroalimentan" (Sosa, 2012, p. 17), es allí donde se estructuran y construyen las relaciones sociales, en la amplitud de su diversidad social, económica, cultural e histórica. Esta diversidad sociocultural es un primer elemento que plantea el desafío y la necesidad de acercarse al territorio y a sus habitantes, para la identificación de elementos de interés cultural, ambiental, social, entre otros, desde la mirada de los propios actores sociales. Bajo esta mirada, se comprenderá el territorio como el resultado de los procesos de la acción social que dan lugar a la apropiación del espacio por parte de los actores sociales de un lugar determinado (Flores, 2007).

Por lo tanto, para la elaboración de la cartografía socioterritorial, se parte de la premisa de que el territorio posee una dimensión social, es decir, es construido socialmente, y por tanto, es diverso. En este caso, se ha considerado la participación social de vecinos y vecinas de la comunidad para identificar y poner en valor aquellos elementos susceptibles de cartografiar. Para Martínez (2012), la dimensión social permite:

Una lectura del territorio de corte histórico, pues los procesos que se dan no son de corto plazo, sino que acumulan experiencias desplegadas por los actores subordinados, muchas de las cuales han sido dejadas de lado, en situaciones en que el campo social estaba dominado por otros intereses (p. 15).

El interés y oportunidad de trabajar directamente con la comunidad local a través de actores sociales del territorio, permite poner en valor la memoria simbólica del espacio, así como el imaginario que han construido a partir de los relatos de sus antecesores y en muchos casos como protagonistas y espectadores de las transformaciones de sus barrios. En consecuencia, con la Universidad liderando esta instancia de construcción colectiva, se generan oportunidades para la inclusión social y participativa de la comunidad identificando elementos de diferentes ámbitos que tienen sentido para su cotidianeidad.

Asimismo, se comprende el territorio como un espacio en donde ocurren trasformaciones, se interrelacionan experiencias, se comparten imaginarios y se reconoce la diversidad. Al respecto Gálvez (2007) considera que:

El espacio es la relación inseparable entre objetos tanto simbólico-artificiales como físico-naturales $y$; relaciones sociales, éstas en todos los tiempos y contextos concretos junto y por medio de un múltiple repertorio de objetos que ellos mismos han creado o dispuesto de la realidad física (p. 8).

En este sentido, es el entramado de significados que los sujetos sociales dan a los elementos de su entorno, materiales o simbólicos, los que van configurando la construcción del espacio y de territorio. Esta construcción social es para cada sujeto 
diferente, lo que permite dimensionar las amplias y diversas miradas que los y las habitantes del cerro tienen respecto de su territorio.

Por otra parte, se define al territorio como un área específica bajo diferentes criterios de ordenamiento territorial. Giménez (1996), define el territorio como "cualquier extensión de la superficie terrestre habitada por grupos humanos y delimitada en diferentes escalas: local, municipal, regional, nacional o supranacional (...) Se trata del espacio estructurado" (p. 10). Considerando lo anterior, para comenzar a planificar el trabajo cartográfico fue necesario delimitar un espacio estructurado a partir de antecedentes políticos y administrativos. Estos márgenes permitieron definir operativamente una zona de trabajo, demarcada por calles, avenidas, quebradas, entre otros.

Se considera importante señalar que la propuesta de comprender el territorio desde la mirada local, a través de sus habitantes, significa poner en valor sus experiencias en torno a sus interpretaciones respecto a elementos que se encuentran en el medio espacial, considerando aspectos subjetivos que forman parte del imaginario simbólico, como la historia narrada de generación en generación en relación al barrio, y otros elementos con los que conviven cotidianamente, como los microbasurales en las calles, que forman parte de la dinámica que deben enfrentar a diario. El hecho de identificar el espacio local y hacerlo parte del territorio, implica comprender la dinámica social de relaciones entre vecinos y vecinas de un mismo barrio, lo cual enriquece la experiencia de generación de conocimiento, permitiendo el encuentro de saberes entre los actores sociales del territorio y la Universidad.

Concebir el territorio como consecuencia de la construcción social que las personas hacen a partir de las interrelaciones sociales con otros sujetos y su entorno, enriquece y transforma el modo en que se describe un territorio determinado, dado que entrega un contexto y un cúmulo de conocimientos que lo hace único. Para Gálvez (2007), la dimensión espacio implica considerar la existencia de procesos sociales y de la historia, los cuales, a partir de las relaciones y acciones sociales que se dan en ese espacio, territorializan ciertas vivencias particulares en un determinado lugar.

De tal forma, el espacio se va a concebir como el lugar donde se construye el territorio, ya que es allí donde se generan los procesos y relaciones sociales, tal como señala Giménez (2005) acerca de que este es "la materia prima a partir de la cual se construye el territorio y, por lo mismo, tendría una posición de anterioridad con respecto a este último" (p. 9).

De este modo, levantar información del territorio desde la institución pública, implica generar diálogo y establecer una relación bidireccional. Al respecto, se pueden distinguir dos formas de conceptualizar y definir el territorio: por un lado existe la mirada político administrativa, donde se definen límites territoriales, por diferentes criterios sujetos al ordenamiento territorial por parte de las autoridades locales, regionales y nacionales. Esta concepción de territorio se contextualiza como territorio 
dado; por el otro, aquella que lo define como un espacio construido por las interrelaciones sociales entre diversos actores sociales que comparten un espacio geográfico determinado (Flores, 2007). Al reconocer la mirada de los actores sociales del territorio se ponen en valor aquellos conocimientos alojados en las comunidades locales, es decir, los saberes que se construyen desde las experiencias en los barrios.

Para comprender mejor de qué manera se teje el territorio como espacio de construcción social, se hace necesaria la referencia a la noción de habitus, la que entrega sentido a las acciones de los actores sociales en un determinado contexto. Al respecto, para Bourdieu (2007), el habitus hace referencia a:

Sistemas de disposiciones duraderas y transferibles, estructuras estructuradas predispuestas para funcionar como estructuras estructurantes, es decir, como principios generadores y organizadores de prácticas y representaciones que pueden estar objetivamente adaptadas a su fin (p. 86).

El habitus, es entendido además como predisposiciones para la acción, y se caracteriza por poseer una estructura simbólica de tipo social, cultural e histórica, que permite dar sentido a las acciones individuales, es decir, las percepciones, los valores y la construcción subjetiva de interpretación de la realidad.

Con este concepto, es posible comprender la relación de los individuos con el espacio social y también geográfico, lugar en donde se da vida a las acciones y desde el cual se estructuran y construyen percepciones respecto al entorno. Por lo tanto, se habla de un territorio construido concebido como un espacio donde confluyen relaciones sociales, se generan vínculos de pertenencia y se construye identidad a partir de las interrelaciones de los actores sociales entorno a un territorio. Al respecto, Giménez (1996) señala que:

El territorio puede ser apropiado subjetivamente como objeto de representación y de apego afectivo, y sobre todo como símbolo de pertenencia socio-territorial. En este caso los sujetos interiorizan el espacio integrándolo a su propio sistema cultural (p.15).

A partir de lo anterior, es posible comprender que en el espacio donde se produce y reproduce la desigualdad social, entendiendo siempre el espacio como construcción social. Al respecto, Bourdieu (1999) distingue entre el espacio físico y el social; este último es de carácter simbólico. Aquí es donde opera la construcción que los sujetos sociales elaboran de otros. Por ello, en un territorio se pueden encontrar diferentes barrios que no sólo están constituidos por las calles, avenidas y geografía, sino además por el imaginario simbólico que deviene de una experiencia, una historia y cultura. Para Bourdieu (1999), "la estructura del espacio se manifiesta, en los contextos más diversos, en la forma de oposiciones espaciales, en las que el espacio habitado (o apropiado) funciona como una especie de simbolización espontánea del espacio social" (p.120). Este sentido de apropiación, determina formas de relaciones sociales en donde las instituciones sociales actúan como agentes reproductores. 
De esta manera, es posible, comprender el sentido de apropiación de los y las habitantes de un barrio con su realidad social, su empatía o indiferencia con otro barrio, aunque ambas formen parte de un mismo territorio. Así, como señala Sosa (2012), "el territorio es objeto de representaciones múltiples, pues múltiples son los actores que desde sus visiones, interpretaciones e intereses le atribuyen determinadas características, potencias, significados" (p.20).

Profundizar sobre espacio y territorio permite relevar la importancia de acercarnos a los actores sociales del barrio, caminar y dialogar mirando las realidades sociales y culturales que se encuentran en un mismo territorio, percibiendo, además, las fronteras tanto simbólicas como geográficas que conforman representaciones socioculturales diferentes tanto para cada individuo como para cada grupo social.

De acuerdo a Barrera (2009), desde las definiciones de espacio y territorio, se identifican dos nociones de espacio: un espacio contenedor vinculado a definiciones administrativas y operativas que representa la porción de tierra que delimita un lugar específico, y por otra parte, el espacio socialmente construido, comprendiendo a este último, como aquel espacio habitado y donde las interrelaciones sociales y los imaginarios sociales que se construyen a partir de la experiencia y lo cotidiano, responden a una realidad subjetiva, es en esta última concepción, donde la cartografía social tiene sentido, en la medida que permite la representación simbólica de los actores sociales con respecto a un determinado territorio.

\section{Cartografía socioterritorial, pasos para un trabajo participativo e inclusivo}

Cuando se habla de una cartografía, sea socioterritorial, social o cultural, habitualmente se asocia a la imagen de un mapa, una mesa y un conjunto de actores sociales dibujando e identificando temas y elementos de interés, para un fin determinado, demandado por una entidad o institución especifica. No obstante, lo principal es señalar que se trata de un proceso participativo e inclusivo, donde la voz de los actores sociales es el principal recurso. En este sentido, para Pérez \& Cano (2012) una cartografía se define como "un medio que nos permite ordenar el pensamiento y generar conocimiento colectivo, nos ubica en lo micro, en el ámbito de las relaciones cotidianas, dentro de un territorio en el que somos y al que hacemos" (p.12). La cartografía social es una herramienta para la generación de conocimientos, donde tanto los saberes locales como los especializados dialogan con un propósito determinado para identificar elementos de valor e interés local que permitan caracterizar el territorio. Para García, Montoya \& Ospina (2014), esta herramienta responde a un "conocimiento dialógico que tiene como fundamento la apertura a formas de conocer y experimentar los territorios que pueden incluso anteceder a los procedimientos lógicos de la representación cartográfica convencional" (p.192).

Los conocimientos que provienen de los saberes locales son incluidos en la construcción cartográfica de manera participativa y colaborativa; de tal modo, los 
mapas y cartografías que se utilizan para recorrer el territorio, son elementos para orientar el trabajo de campo y no un determinante para caracterizar el territorio. Es una herramienta que permite la construcción de conocimiento desde la realidad social de los actores locales.

De este modo, la cartografía actúa como herramienta para el levantamiento de información, representando una instancia reflexiva que lleva a los actores sociales a mirar su entorno, poner atención a lo cotidiano y a lo vivido; invita a la reconstrucción de las experiencias vividas y a un recorrido por la historia, poniendo en valor temas de interés y otros de conflicto. En este sentido, para Varela (2012), la cartografía es una "oportunidad para la enunciación y sistematización de conocimientos locales sobre el espacio habitado" (p. 70), constituyéndose de esta manera en una poderosa herramienta metodológica de construcción de conocimiento de carácter participativo e inclusivo, donde los diferentes puntos de vista reflejan las dinámicas sociales y culturales sobre un espacio determinado.

Desde una mirada más amplia, también la cartografía se concibe como una herramienta de consulta para orientar las acciones tanto de la comunidad local como universitaria y por lo mismo, un espacio de generación de información en permanente construcción, donde diferentes personas puedan aportar información respecto de su barrio de acuerdo a los procesos y trasformaciones territoriales. El territorio es el espacio donde se desarrollan las transformaciones sociales, dinámicas y prácticas sociales, por lo que el trabajo en terreno y la participación de actores locales, implica reconocer y poner en valor estos procesos colectivos que responden a una realidad social determinada.

La importancia de la cartografía como herramienta, permite dar sentido a dinámicas sociales que son heterogéneas en la amplitud del territorio. Como señalan Pérez \& Cano (2012), "la cartografía social parte de lo cotidiano, de lo simple, de lo común a todos, para ir avanzando hacia lo complejo de las relaciones humanas, en vínculo e interacción con el territorio en el que se desarrollan, que las implica y al que construye a la vez" (p.13).

\section{Metodología empleada en la experiencia desarrollada en el territorio de Playa Ancha}

El barrio es el espacio donde los sujetos sociales realizan su vida cotidiana y colectiva más cercana. Es en éste donde se desarrolla el sentido de pertenencia e identidad. Asimismo, posee atributos únicos vinculados a la geografía, a la estructura espacial, las prácticas sociales, entre otros, que son reconocidos por sus habitantes. La Mesa Territorial de Desarrollo, instancia anteriormente descrita, busca la integración de los actores sociales para lograr fortalecer el tejido social y así mejorar la calidad de vida de los y las habitantes a través del desarrollo territorial. Se requiere el reconocimiento y la identificación de los elementos presentes en el territorio que permitan una 
caracterización del cerro, es por este motivo que se propuso microzonificar el territorio de Playa Ancha, para graficarlo en una cartografía socioterritorial, que permitiera proporcionar recursos de información para la toma de decisiones en torno al desarrollo del cerro.

Como todo proceso llevado a cabo desde el Convenio de Desempeño (UPA 1301), que ha implicado relación y acciones en el territorio, se presentó una propuesta de construcción cartográfica a la Mesa Territorial Desarrollo, donde se manifestó a partir de la iniciativa de los propios actores sociales, la necesidad de mayor información en temas tales como: medioambiente, zonas de contaminación, espacios de acumulación de basuras de forma periódica, sitios eriazos, sitios de reciclaje, espacios de valor histórico y patrimonial, paisaje y entorno público, espacios de reunión y participación, centros de salud pública, entre otros.

Para llevar a cabo lo anterior, fue necesario identificar y delimitar los márgenes del trabajo, lo que también significó reconocer las capacidades y alcances para acceder a ciertos sectores del territorio, considerando las características geográficas del cerro, especialmente sus quebradas y acantilados.

Se estableció trabajar en base a los límites del cerro Playa Ancha planteados en la Encuesta Socioterritorial llevada a cabo el año 2014 por el Observatorio de Innovación Social, en donde el cerro se dividió operativamente en 4 macrozonas. Es preciso recordar que Playa Ancha posee una población cercana a los 61.460 habitantes y una superficie de $6,1 \mathrm{~km}^{2}$, por lo que la división por macrozonas permitió ordenar el trabajo de campo.

Estas macrozonas se dividieron en sub-macrozonas o microzonas para lograr identificar elementos con mayor detalle. El propósito consistió en recorrer barrios o microzonas junto con actores sociales claves de la MTD y el equipo del Observatorio de Innovación Social, identificando elementos mediante el diálogo, la conversación y la interacción con otros vecinos o vecinas que eran invitados durante el recorrido por los propios actores claves.

Cabe añadir, que establecer límites para definir un territorio se sustenta en requerimientos operativos para levantar acciones específicas. Para la cartografía, la delimitación responde a fines técnicos con el fin de delimitar el trabajo de campo, esto no significa que para el imaginario simbólico de cada habitante, colectivo, población, barrio o grupo social, sean esos los límites territoriales que definen el cerro Playa Ancha, ya que éste puede ser concebido como una porción territorial más grande o más pequeña. También es necesario recordar la cantidad de cerros que componen la ciudad de Valparaíso y la conurbación que existe entre estos.

Además, que dadas las características geográficas y espaciales de Playa Ancha y desde una perspectiva metodológica, se realizaron dos experiencias pilotos. La primera se desarrolló en el barrio de Puertas Negras y la segunda en la Avenida Playa Ancha, barrios opuestos en su arquitectura, urbanización e historia, lo que permitió 
comprender la necesidad de flexibilizar la pauta de trabajo utilizada en terreno y desarrollada a partir de una reflexión inicialmente teórica. Factores como el viento, la seguridad en el espacio público, el comercio y las realidades socioculturales en general, indicaron los elementos, materiales y etapas para un adecuado trabajo de campo.

Una vez analizada la experiencia piloto, se categorizaron elementos de interés levantados en el territorio y luego se digitalizó la información en un software especializado. Así en esta experiencia, se pudo determinar de qué manera se planificarían las siguientes salidas a terreno, para lo cual se estimaron un total de 28 salidas al territorio para levantar información conjunta entre el equipo del Observatorio de Innovación Social y actores sociales de la Mesa Territorial de Desarrollo, estableciéndose que, para cada Macrozona (4), se considerasen siete microzonificaciones, por lo que el proceso en terreno y de digitalización de la información tuvo una duración de siete meses.

Se elaboró una tabla con categorías para el estudio, que incluyó elementos medioambientales, sitios de valor histórico y patrimonial, paisaje y entorno público, espacios de reunión y participación, centros de salud pública, entre otras, y cada una de éstas se subdividió en subcategorías. Lo anterior, no limitó la construcción de nuevas categorías que surgieron durante el trabajo de campo relacionados con la realidad social de cada barrio.

En relación a lo anterior, los pasos o etapas que conformaron la construcción de la cartografía social, fueron los siguientes:

-Planificación y coordinación salidas a terreno: se estimó un calendario que permitió coordinar y planificar la participación de cada actor clave y la participación de otros vecinos y vecinas que se motivaron a participar. En un período de siete meses se realizaron dos salidas a terreno cada semana, considerando que algunos recorridos por el barrio tenían una duración aproximada de dos horas y que el factor climático fue importante de considerar.

-Identificación de cada macrozona y microzonas a recorrer: esto significó la generación de mapas, identificación de calles y posibles rutas a sugerir, previas a la salida de terreno. Existieron coordinaciones con actores claves de los sectores a recorrer, determinándose una zona de influencia aproximadamente de 80 a $200 \mathrm{mt}^{2}$, por cada salida a terreno.

-Trabajo de campo: antes de iniciar el recorrido en conjunto con el actor clave, se cotejó el nombre de direcciones de calles y de la realidad espacial del territorio. Lo anterior, en el contexto de que el nombre de algunas calles responde al imaginario cotidiano de los y las habitantes del barrio, y no al nombre establecido desde la institucionalidad administrativa de la ciudad. Se complementó la información obtenida, 
con un registro fotográfico, además, con un GPS, el mapa respectivo de cada sector y una tabla de atributos o categorías cartográficas.

-Inicio del recorrido por el barrio: como se mencionó anteriormente los recorridos tuvieron una duración de aproximadamente dos horas, pero también el tiempo fue relativo a diversos factores, como el clima y disponibilidad del actor clave, como ya se señaló. En este mismo proceso, se sumaron algunos vecinos y vecinas que espontáneamente quisieron participar de la cartografía.

-Digitalización de la información e incorporación de nuevas categorías en el caso de que hayan surgido.

-Validación de la información: la última etapa, corresponde a la validación de información, corregir y/o agregar aspectos y elementos de interés. Este proceso fue de carácter participativo a través del trabajo en la Mesa Territorial de Desarrollo y la generación de mapas con contenido cartográfico levantado.

-Actualización de la información: se ha considerado como un proceso permanente que surge no sólo de la validación de la información, sino también de nuevos aportes desde los y las habitantes del cerro, así como de la comunidad universitaria.

Con respecto al equipo que participó de la cartografía, se contó con la coordinación de la investigadora responsable del Observatorio, una encargada del trabajo de campo y de la digitalización, y los asistentes del trabajo en terreno; estos tres últimos, estudiantes de la Universidad del área de geografía, actores claves participantes de la Mesa Territorial de Desarrollo, vecinos y vecinas de los barrios.

De este modo, la cartografía socioterritorial responde a un trabajo en conjunto con la comunidad del cerro y la Universidad, un esfuerzo por generar procesos colaborativos y construcción colectiva de conocimiento, para la caracterización del espacio habitado y la generación de acciones que permitan mejorar la calidad de vida de sus habitantes.

\section{Conclusiones}

Considerando el trabajo presentado, se señala la importancia que tiene repensar la lógica de las relaciones desde las instituciones públicas con el territorio y sus comunidades, lo cual es un desafío que las universidades deben asumir. La posibilidad de generar instancias para el diálogo y las sinergias orientadas al desarrollo territorial y 
la calidad de vida de los y las habitantes, deben ser la guía que oriente la generación de conocimiento, y para que esto sea posible, es necesario instalar espacios para la inclusión social de los actores sociales en las comunidades locales.

Uno de los grandes desafíos actuales del quehacer de las universidades estatales, tiene relación con la vinculación con su entorno, local, regional y también nacional, es decir, con la generación de alianzas entre la universidad y el territorio para la generación de conocimiento compartido que se oriente a mejorar la calidad de vida de las personas. Se pretende que este conocimiento tenga incidencia en los territorios y en los procesos de transformación de éstos. Es en este contexto, donde la Universidad, a través del Convenio de Desempeño: "Un Modelo Replicable de Innovación Social para el Desarrollo Territorial de Playa Ancha" y a través de la cartografía socioterritorial, intenta tomar un rol transformador haciendo partícipes de manera inclusiva a los actores sociales del territorio.

La construcción de conocimiento colectivo en torno al espacio habitado, a través de la cartografía social, pone en valor las dinámicas sociales de lo cotidiano y releva el habitus de quienes habitan un espacio determinado, asegurando el levantamiento de información pertinente para el mejoramiento y desarrollo de la calidad de vida de los y las habitantes del territorio. Concebir el concepto de habitus, tal como señaló Bourdieu (2007), pone de manifiesto el conjunto de prácticas y conocimientos que configuran la cultura, los modos de vida y cosmovisión de las personas. En el trabajo de la cartografía socioterritorial, se consideró el habitus como esquema sistemático de prácticas sociales que han sido y son parte de la vida de los y las habitantes, las que inciden en la forma de entender el espacio y relacionarse con el entorno.

Al respecto, Bourdieu (1999) y Giménez (1996), señalan que existe una concepción simbólica, la que es construida socialmente por los sujetos sociales, dotando de sentido de pertenencia y apropiación hacia el espacio habitado. Lo anterior, permite comprender la diversidad de miradas y experiencias que se cruzan al identificar y trazar en la cartografía socioterritorial aquellos ámbitos significativos que permiten a sus habitantes una mejor relación con su entorno.

De la experiencia de la cartografía socioterritorial, se puede señalar que, la dimensión social del territorio devela los imaginarios sociales y la memoria simbólica que albergan las comunidades, representa los procesos sociales y los modos de vida que se llevan a la práctica en los territorios. Asimismo, implica considerar los procesos históricos y socioculturales de quienes habitan ese espacio, puesto que son el resultado de las percepciones y formas de interrelación que se han construido socialmente. 
La cartografía como metodología se enriquece al poseer en sus contenidos la mirada socio espacial y simbólica que los y las habitantes construyen sobre el territorio. Asimismo, la experiencia descrita, refleja el diálogo de saberes que se ponen a disposición entre la universidad y el territorio, permitiendo el abordaje de diferentes temáticas disciplinares que pueden ser sustentadas por la universidad en pro del compromiso público y con el territorio. Como señalan Pérez y Cano (2012), la cartografía socioterritorial ha permitido la generación de conocimiento colectivo, donde no sólo ha tenido cabida el discurso y miradas de quienes habitan el territorio, sino también estudiantes y profesionales, haciendo posible el acercamiento hacia la comunidad de forma dialogante, facilitando procesos de relaciones sociales horizontales.

De esta manera, se considera que, generar instancias para el diálogo bidireccional y horizontal, posibilita procesos transformadores, que permiten la puesta en valor de los diferentes saberes, académicos, vivenciales, técnicos y muchos más, que al canalizarse en un propósito, como el desarrollo territorial, tiene efectos positivos que refuerzan la confianza entre la institución pública y la comunidad.

Un ámbito importante que se debe destacar es cómo este tipo de prácticas también son transformadoras en la formación de los y las estudiantes. Se señala en relación a ello que para la elaboración de la cartografía socioterritorial, estudiantes del área de geografía pusieron en práctica sus conocimientos, desde un enfoque social e inclusivo, complementando sus saberes especializados con los conocimientos locales.

La cartografía socioterritorial como experiencia de innovación social, se enmarca en la generación de conocimiento compartido; es un proceso que tiene como producto describir el territorio y orientar la toma de decisiones en torno a actividades que favorezcan el desarrollo territorial, en el cual participan diferentes miradas en el proceso de levantamiento de información. La experiencia desarrollada en la cartografía socioterritorial, formó parte de un trabajo colaborativo que evidencia procesos de innovación e inclusión social en la construcción de conocimiento entre la Universidad y el territorio, que se sustentó a partir de principios de confianza entre la institución pública y los actores locales, a partir de diálogo horizontal y colaborativo.

El trabajo realizado es un referente para pensar y re-pensar la vinculación con el territorio, sus habitantes y su entorno, ya que implicó reflexionar sobre la diversidad social y cultural, las historias y experiencias propias de los actores sociales en sus barrios, sus imaginarios sociales y las dinámicas sociales de la cotidianeidad. Una posibilidad que permitió conocer en la práctica el territorio de Playa Ancha y la realidad social del cerro más grande de la ciudad de Valparaíso. 


\section{Bibliografía}

Aguirre, B. \& Rabi, S. (2009). La trayectoria espacial de la Corporación de la Vivienda (CORVI). Revista Electrónica DU\&P. Diseño Urbano y Paisaje Volumen VI. 18, 1-73. Recuperado de: http://www.ucentral.cl/du\&p/pdf/18 trayectoria espacial b.pdf

Barrera, S. (2009). Reflexiones sobre Sistemas de Información Geográfica Participativos (SIGP) y cartografía social. Revista Colombiana de Geografía, 18, 923. Recuperado de: http://www.redalyc.org/articulo.oa?id=281822001002

Bourdieu, P. (1999). La miseria del mundo. Madrid: Akal.

Bourdieu, P. (2007). El sentido práctico. Buenos Aires: Siglo XXI.

Flores, M. (2007). La identidad cultural del territorio como base de una estrategia de desarrollo sostenible. Revista Opera, 7, 35-54. Recuperado de: http://www.redalyc.org/pdf/675/67500703.pdf

Gálvez, E. (junio 2014). El concepto de Habitus y su dimensión espacial: apuntes para un habitus territorial y regional. En: Memorias Primer Congreso Latinoamericano de Estudiantes de Posgrado en Ciencias Sociales (CLEPSO). Recuperado de: http://clepso.flacso.edu.mx/sites/default/files/clepso.2014 eje 8 galvez.pdf

García, A., Montoya, V. \&, Ospina, C. (2014). Andar dibujando y dibujar andando: cartografía social y producción colectiva de conocimientos. Revista Nómadas, 40, 190-205. Recuperado de: http://www.redalyc.org/articulo.oa?id $=105131005013$

Giménez, G. (1996). Territorio y cultura. Revista Estudios sobre las Culturas Contemporáneas, $4, \quad 9-30.4$ Recuperado de: http://www.redalyc.org/pdf/316/31600402.pdf

Giménez, G. (2005). Territorio e identidad, Breve introducción a la geografía cultural. Revista Trayectorias, 17, 8-24. Recuperado de: http://www.redalyc.org/articulo.oa?id=60722197004

Ilustre Municipalidad de Valparaíso. (2016). Una Síntesis de lo que somos. Recuperado de:http://www.municipalidaddevalparaiso.cl/valparaiso sintesis.aspx 
Martínez, L. (2012). Apuntes para pensar el territorio desde una dimensión social. Revista Ciências Sociais Unisinos, 1, 12-18. Recuperado de: http://www.redalyc.org/articulo.oa?id $=93823702003$

Pérez, M \& Cano, A. (2012). Laboratorio barrial de experiencias. Programa Integral Metropolitano. Uruguay: Universidad de la República.

Sosa, M. (2012). ¿Cómo entender el territorio? Guatemala: Cara Parens.

Varela, D., Vélez, I. \& Rátiva, S. (2012). Cartografía social como metodología participativa y colaborativa de investigación en el territorio afrodescendiente de la cuenca alta del río Cauca. Revista Colombiana de Geografía, 2. 59-73. Recuperado de: http://www.redalyc.org/articulo.oa?id=281823592005

Universidad de Playa Ancha. (2014). Convenio de Desempeño UPA 1301. Huellas de Playa Ancha. Historias de su poblamiento. Valparaíso: Universidad de Playa Ancha.

Universidad de Playa Ancha. (2015). Convenio de Desempeño UPA 1301. Encuesta Socioterritorial de Playa Ancha. Informe de resultados. Valparaíso: Universidad de Playa Ancha. 